\title{
Calculation of the Energy Band Diagram of a Photoelectrochemical Water Splitting Cell
}

Peter Cendula,,+ S. David Tilley, ${ }^{\ddagger}$ Sixto Gimenez, " Juan Bisquert, " Matthias

Schmid, ${ }^{\dagger}$ Michael Grätzel, ${ }^{\ddagger}$ and Jürgen O. Schumacher ${ }^{\dagger}$

Institute of Computational Physics, Zurich University of Applied Sciences (ZHAW),

Wildbachstrasse 21, 8401 Winterthur, Switzerland, Laboratory of Photonics and Interfaces, Ecole Polytechnique Fédérale de Lausanne, EPFL-SB-ISIC-LPI, Station 6, 1015 Lausanne, Switzerland, and Photovoltaics and Optoelectronic Devices Group, Departament of Physics, University Jaume I, 12071 Castellon, Spain

E-mail: cend@zhaw.ch

${ }^{*}$ To whom correspondence should be addressed

†Zurich University of Applied Sciences

${ }^{\ddagger}$ Ecole Polytechnique Fédérale de Lausanne

ฯUniversity Jaume I 


\begin{abstract}
A physical model is presented for the semiconductor electrode of a photoelectrochemical (PEC) cell. The model accounts for the potential drop in the Helmholtz layer and thus enables description of both band edge pinning and unpinning. The model is based on the continuity equations for charge carriers and direct charge transfer from the energy bands to the electrolyte. A quantitative calculation of the position of the energy bands and the variation of the quasi-Fermi levels in the semiconductor with respect to the water reduction and oxidation potentials are presented. Calculated photocurrent-voltage curves are compared with established analytical models and experimental data. Our model calculations are suitable to enhance understanding and improve the properties of semiconductors for photoelectrochemical water splitting.
\end{abstract}

Introduction Research on hydrogen production with photoelectrochemical (PEC) cells is propelled by the worldwide quest for capturing, storing and using solar energy instead of the decreasing fossil energy reserves. Hydrogen is widely considered as a key solar fuel of the future. ${ }^{1}$ Hydrogen is also part of power-to-gas conversion systems developed to resolve the intermittency in wind and solar energy production. ${ }^{2}$ Although a PEC/photovoltaic cell with $12.4 \%$ efficiency was demonstrated with $\mathrm{GaInP}_{2} / \mathrm{GaAs}^{3}{ }^{3}$ decreasing its cost and increasing its lifetime remain a challenge. An alternative approach often pursued is to use abundant and cheap metal oxides as the semiconductor materials for PEC electrodes. ${ }^{4-6}$ However, their recombination losses, charge carrier conduction and water oxidation properties need to be understood and optimized both by measurement and numerical simulation in order to further advance these materials. ${ }^{7}$

Several approaches for a mathematical analysis of semiconductor electrodes can be found in the literature, including analytical ${ }^{8,9}$ and numerical models ${ }^{10,11}$ of PEC cells. An extensive numerical study of PEC behavior of $\mathrm{Si}$ and $\mathrm{GaP}$ nanowires was recently conducted with commercial software. ${ }^{12}$ Since surface states play a major role for many semiconductors, corresponding models were also developed to analyze their effect on the electrochemical 
measurements. ${ }^{13-15}$ On the PEC system level, models of the coupled charge and species conservation, fluid flow and electrochemical reactions were recently developed. ${ }^{16,17}$ The latter studies revealed how PEC systems should be designed with minimal resistive losses and low crossover of hydrogen and oxygen by use of a non-permeable separator.

Almost every publication on PEC cells features a schematic energy band diagram of a PEC cell, mostly sketched by hand from basic physical understanding described in textbooks on electrochemistry. ${ }^{7,18,19}$ Although such sketches might be qualitatively correct, numerical calculations of the charge carrier transport might reveal additional features not captured by the sketches. We are aware that the development of numerical calculations is frequently hindered by the complicated physical processes in the actual materials and lack of measurements of parameter values for these processes. ${ }^{20}$ In spite of these obstacles, we think that the recent advent of user-friendly numerical software and advanced measurement techniques could fill the gap between the experimental and numerical approaches.

Model In this work, we present calculations of the energy band diagram of a PEC electrode from a physical model with clearly formulated assumptions. ${ }^{21}$ The model is based on charge carrier continuity equations with direct charge transfer from the valence or conduction band to the electrolyte. We consider a PEC cell consisting of a thin compact n-type semiconductor, hence PEC cells with porous structures ${ }^{22,23}$ are not directly described with our model. We assume an electrolyte that can easily accept a single electron or hole (such as $\mathrm{H}_{2} \mathrm{O}_{2}{ }^{24}$ or $\left.\left[\mathrm{Fe}(\mathrm{CN})_{6}\right]^{3-/ 4-25}\right)$. Charge transfer occurs across the semiconductor/electrolyte interface until an equilibrium charge distribution is reached and the equilibrium Fermi level in the semiconductor $E_{F 0}$ becomes equal to the redox Fermi level $E_{r e d o x}$

$$
E_{F 0}=E_{\text {redox }} .
$$

We reserve subscript 0 for equilibrium values in the dark in the following. To derive our model, we use and repeat some of the general definitions introduced in our previous work ${ }^{26}$ 
to describe the PEC cell in steady-state under illumination (out of equilibrium), Figure 1 and Table 1. Note that we use a notation of subscript $s c$ for semiconductor, $s$ for surface quantity, $b$ for a bulk semiconductor quantity (where electrons and hole remain at equilibrium in the dark).

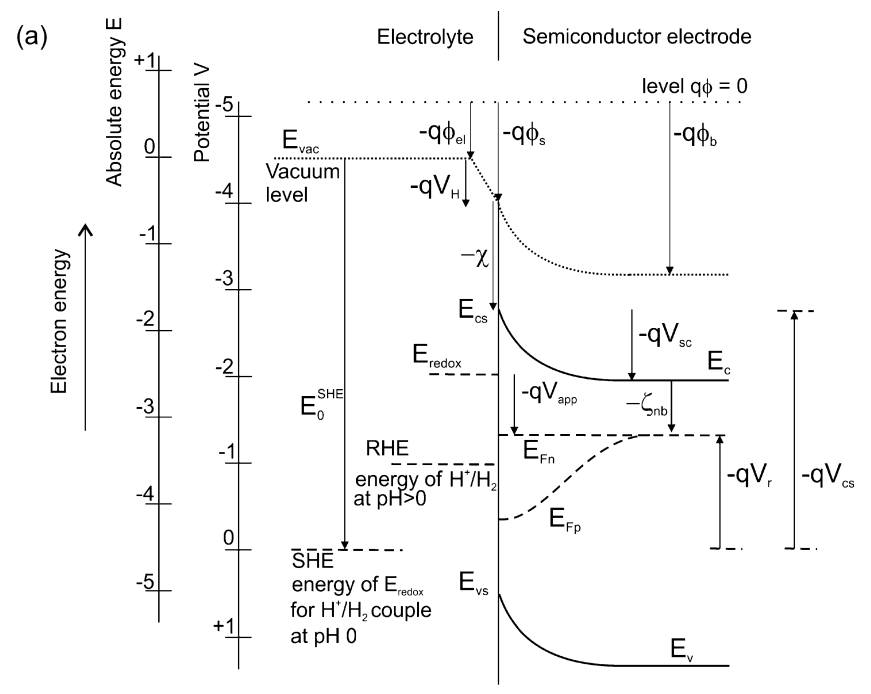

Figure 1: Scheme of an n-type semiconductor electrode in steady-state under illumination, with electron energy indicated in the absolute energy scale (with respect to the vacuum level), and potentials in the electrochemical scale, with respect to SHE. Reprinted with permission from Ref. 26. Copyright (2014) American Chemical Society.

Bulk equilibrium properties of the isolated semiconductor are denoted with a subscript $0 i$. The bulk of the semiconductor is electrically neutral, hence the concentration of electrons in the bulk $n_{0 i}$ must be equal to the number of fully ionized donors $N_{D}, n_{0 i}=N_{D}$ (for $\left.N_{D} \gg n_{i}\right)$. Thus, the concentration of holes is $p_{0 i}=n_{i}^{2} / n_{0 i}$, where $n_{i}$ denotes intrinsic carrier concentration. An isolated unbiased semiconductor before contact to an electrolyte has a conduction band edge $E_{c, 0 i}$ and a Fermi level $E_{F, 0 i}$ related to the vacuum level $E_{v a c}$ and to the electron affinity $\chi$ by

$$
\begin{aligned}
E_{c, 0 i} & =E_{v a c}-\chi \\
E_{F, 0 i} & =E_{c, 0 i}-\zeta_{n b}, \\
\zeta_{n b} & =k_{B} T \ln \left(\frac{N_{C}}{n_{0 i}}\right),
\end{aligned}
$$


where $k_{B}$ is the Boltzmann constant, $T$ is the temperature, $q$ is the elementary charge and $N_{C}$ is the effective density of states in the conduction band, and $\zeta_{n b}$ is the distance of the conduction band edge to the Fermi level. In the following, we use $E_{v a c}=0 \mathrm{eV}$ as is the convention. The potential drop in the Helmholtz layer in the dark $V_{H}$ is calculated from the local vacuum level (LVL) at the surface of the semiconductor $\left(-q \phi_{s}\right)$ and the LVL of the electrolyte $\left(-q \phi_{e l}\right)$, Figure 1,

$$
-q V_{H}=-q \phi_{s}-\left(-q \phi_{e l}\right)
$$

Note that the potential drop in the Helmholtz layer can be a different value at flatband situation (denoted $V_{H}^{f b}$ ) than at the other measured voltage (denoted $V_{H}$ ). We measure the voltage $V_{r}$ of the semiconductor electrode with respect to a reference electrode, which is the difference of the Fermi level of electrons in the semiconductor back contact $E_{F n, b}$ and the Fermi level of the reference electrode $E_{0}^{S H E}$

$$
V_{r}=-\frac{E_{F n, b}-E_{0}^{S H E}}{q} .
$$

In this article, we use both the standard hydrogen electrode (SHE) energy and the reversible hydrogen electrode (RHE) as reference electrodes and scale of energy. The measured voltage with respect to the SHE is denoted $V_{r}$ (without subscript SHE) and the measured voltage with respect to the RHE $V_{r, R H E}$ with

$$
V_{r, R H E}=V_{r}+2.3 V_{t h} \cdot p H
$$

where $V_{t h}=\frac{k_{B} T}{q}$ is the thermal voltage and $p H$ denotes the $\mathrm{pH}$ value of the solution. We draw attention to the fact that negative bias versus RHE brings the energy closer to the vacuum level $E_{v a c}$. The position of the electron Fermi level at the semiconductor back 
contact is calculated as (see Figure 1)

$$
E_{F n, b}=-q V_{H}-\chi-q V_{s c}-\zeta_{n b},
$$

where $V_{s c}$ denotes the potential drop in the semiconductor. What is usually reported in the literature is the value of the flatband potential, which is the measured voltage when the bands are flat $\left(V_{s c}=0\right)$

$$
V_{f b}=\left.V_{r}\right|_{V_{s c}=0}=\frac{E_{0}^{S H E}+\chi+\zeta_{n b}}{q}+V_{H}^{f b}
$$

The value of $V_{H}^{f b}$ is often not known as it depends on the surface conditions of the semiconductor in the electrolyte. For this article, we use the known values of $V_{f b}$ and $\chi$ and we determine $V_{H}^{f b}$ from eq. 9. The potential drop in the semiconductor $V_{s c}$ can be expressed from Figure 1 as

$$
-q V_{s c}=-q \phi_{b}-\left(-q \phi_{s}\right)
$$

Then from eqs. 6, 8, 9 follows

$$
V_{s c}=V_{r}-V_{f b}-\left(V_{H}-V_{H}^{f b}\right)
$$

The second option is to refer the voltage to the equilibrium of the semiconductorelectrolyte interface (SEI) and this value is denoted $V_{a p p}{ }^{26}$

$$
\begin{aligned}
& V_{a p p}=-\frac{E_{F n, b}-E_{r e d o x}}{q}, \\
& V_{a p p}=V_{s c}-V_{b i}+V_{H}-V_{H 0},
\end{aligned}
$$

where the built-in voltage is denoted $V_{b i}$ and the potential drop across the Helmholtz layer in the dark equilibrium $V_{H 0}$. The equilibrium of SEI means $V_{a p p}=0 \mathrm{~V}$.

On the semiconductor side of the junction, the electrostatic potential $\phi$ is obtained by 
solving Poisson's equation ${ }^{19}$

$$
\frac{d^{2} \phi}{d x^{2}}=-\frac{q\left(N_{D}-n(x)+p(x)\right)}{\varepsilon_{0} \varepsilon_{r}},
$$

where $\varepsilon_{0}$ is the permittivity of vacuum, $\varepsilon_{r}$ is the relative permittivity of the semiconductor, $N_{D}$ is the concentration of fully ionized donors, $n(x)$ is the concentration of free electrons and $p(x)$ is the concentration of free holes $(p(x) \ll n(x)$ for n-type semiconductor in the dark). We can write for the conduction and the valence band edge energies $E_{c}$ and $E_{v}$ in the electrostatic potential $\phi(x)$

$$
\begin{aligned}
& E_{c}(x)=-\chi-q\left(\phi(x)-\phi_{e l}\right), \\
& E_{v}(x)=E_{c}(x)-E_{g}
\end{aligned}
$$

where bandgap energy of the semiconductor is $E_{g}$. Band edge pinning (constant value of $E_{c s}$ and $E_{v s}$ for any measured voltage) is present if $V_{H}=V_{H}^{f b}$ for any measured voltage, otherwise the band edges become unpinned (values of $E_{c s}$ and $E_{v s}$ vary with measured voltage).

A simple approximation to solve Poisson's equation, Eq. 14, is to assume that the total space charge is uniformly distributed inside the space charge region (SCR) of width $w$ (also called the depletion region approximation)

$$
w=\sqrt{\frac{2 \varepsilon_{0} \varepsilon_{r}}{e N_{D}}\left|V_{s c}\right|} .
$$

The boundary conditions for the electrostatic potential $\phi$ follow directly from the definitions in Figure 1

$$
\begin{aligned}
\phi(0) & =\phi_{s}, \\
\phi(w) & =\phi_{b} .
\end{aligned}
$$


The concentration of free electrons and holes in the dark $n_{\text {dark }}(x)$ and $p_{\text {dark }}(x)$ can be written as

$$
\begin{aligned}
& n_{\text {dark }}(x)=n_{0 i} \exp \frac{\frac{\phi(x)-\phi_{b}}{V_{t h}}}{}, \\
& p_{\text {dark }}(x)=p_{0 i} \exp \frac{\frac{-\phi(x)+\phi_{b}}{V_{t h}}}{} .
\end{aligned}
$$

The value of the electrostatic potential in the semiconductor bulk $\phi_{b}$ appears in the above expressions because we have made a general definition of the electrostatic potential including the potential drop in the Helmholtz layer. Therefore, $\phi_{b}$ is not zero, in contrast to a recent textbook definition. ${ }^{7}$ The approximate solution of Poisson's eq. $\phi_{a}$ is then

$$
\begin{aligned}
& \phi_{a}(x)=\phi_{b}-\operatorname{sign}\left(V_{s c}\right) \frac{q N_{D}}{2 \varepsilon_{0} \varepsilon_{r}}(w-x)^{2}, \quad 0<x<w \\
& \phi_{a}(x)=\phi_{b}, \quad w<x<d .
\end{aligned}
$$

When the measured voltage $V_{r}$ is positive of the flatband potential $V_{f b}$, the n-type semiconductor is in the depletion regime. When the measured voltage is negative of the flatband potential, the semiconductor is in the accumulation regime (due to the sign of $V_{s c}$ ).

In the following analysis, we neglect reflection losses and absorption in the electrolyte. Both illumination directions from electrolyte-electrode (EE) or substrate-electrode (SE) are included with the generation rate of charge carriers given by the simple Lambert-Beer law $G_{h}(x)=\alpha P e^{-\alpha x}$ for EE illumination or $G_{h}(x)=\alpha P e^{-\alpha(d-x)}$ for SE illumination. The number of photons with energy above $E_{g}=\frac{h c}{\lambda_{g}}$ that are absorbed in the semiconductor is $P=\int_{\lambda_{\min }}^{\lambda_{g}} \Phi(\lambda) d \lambda$, the spectral photon flux of standard AM1.5G spectrum with intensity $100 \mathrm{~mW} / \mathrm{cm}^{227}$ is $\Phi(\lambda)$ and the absorption coefficient of the semiconductor is $\alpha$. We assume low-injection conditions with the number of photogenerated electrons smaller than the donor concentration. Hence, the electron concentration is roughly equal to the dark electron concentration $n(x)=n_{\text {dark }}(x)$. The hole continuity equation is solved to obtain the free hole 
concentration $p$ inside of the semiconductor of thickness $d$

$$
0=-\frac{1}{q} \frac{\partial j_{h}}{\partial x}+G_{h}(x)-R_{h}(x)
$$

The hole current density $j_{h}$ is expressed using the analytical solution of Poisson's equation

$$
j_{h}=-q D_{h} \frac{\partial p}{\partial x}-q \mu_{h} p \frac{\partial \phi_{a}}{\partial x}
$$

where $\mu_{h}=\frac{q D_{h}}{k_{B} T}$ is the hole mobility, and $D_{h}$ is the hole diffusion constant. Direct band-toband nonlinear recombination is assumed

$$
R_{h}=\frac{1}{N_{D} \tau_{h}}\left(n_{\text {dark }} p-n_{i}^{2}\right)
$$

We assume that charge transfer under illumination occurs exclusively from the valence band to the electrolyte. We do not include charge transfer from surface states in the current analysis. The current density of valence band holes at the SEI is described by a first-order approximation $^{28}$

$$
j_{h}(0)=-q k_{t r h}\left(p(0)-p_{d a r k}(0)\right)
$$

where $k_{t r h}$ is the rate constant for hole transfer, and a linear dependence on the difference of the interfacial hole concentration $p(0)$ from its dark value $p_{\text {dark }}(0)$ at the interface is assumed. Since the thickness of the semiconductor is in the order of the penetration length of light $\alpha^{-1}$ for the hematite parameters listed in Table 2, we consider the hole current at the back contact of the semiconductor to depend on a surface recombination velocity $r_{s}$

$$
j_{h}(d)=+q r_{s}\left(p(d)-p_{0 i}\right) .
$$


We use $r_{s}=10^{5} \mathrm{~m} / \mathrm{s}$ for numerical calculations throughout this article. ${ }^{12} \mathrm{In}$ order to obtain convergence of the numerical solution procedure, the continuity equation was solved in a nondimensional form after applying the usual normalization of the variables of the drift-diffusion equations. ${ }^{29}$

The quasi-Fermi energies $E_{F n}, E_{F p}$ under the influence of an electrostatic potential $\phi(x)$ are calculated by the Boltzmann distribution

$$
\begin{aligned}
& n(x)=N_{C} \exp \left(-\frac{E_{c}(x)-E_{F n}}{k_{B} T}\right), \\
& p(x)=N_{V} \exp \left(-\frac{E_{F p}-E_{v}(x)}{k_{B} T}\right) .
\end{aligned}
$$

Results and Discussion We numerically solved the hole (electron) continuity equation Eq. 22 for an n-type (p-type) semiconductor by using the depletion region approximation of the electrostatic potential Eq. 21. The results upon EE illumination for the n-type $\mathrm{Fe}_{2} \mathrm{O}_{3}$ and the p-type $\mathrm{Cu}_{2} \mathrm{O}$ are presented in the following. If not otherwise stated, we assume $\phi_{e l}=0 \mathrm{~V}$ and band edge pinning $\left(V_{H}^{f b}=V_{H 0}=V_{H}\right)$ in the following.

Our work for simplicity does not include the effect of electrocatalyst on the photoelectrode surface, even though its use is undoubtably necessary for practical devices. ${ }^{30}$ Recently, a numerical model to describe realistic electrocatalysts with various porosity and ion-permeability appeared. ${ }^{31}$ The main conclusion of Ref. 31 is that the adaptive and metallic catalyst differ mainly with respect to the (electrostatic) potential drop. For the adaptive catalyst the potential drop develops only in the semiconductor, whereas for the metallic catalyst the potential drop develops both in the electrolyte and in the semiconductor. Our model already assumes that the potential drop is prescribed as a material/interface parameter, hence both adaptive and metallic catalyst of Ref. 31 can be included in our model by defining the value of this potential drop. 
$\mathbf{F e}_{2} \mathrm{O}_{3}$ The charge carrier concentration profiles calculated from the model are plotted in Figure 2. In the dark, the SCR is depleted of electrons and the concentration of holes is larger than the bulk hole concentration. For increasing $V_{r, R H E}$, the dark electron concentration at the SEI $n_{\text {dark }}(0)$ decreases until it is smaller than the dark hole concentration at the SEI $p_{\text {dark }}(0)$, leading to an inversion layer characterized by larger concentration of holes (minorities) than electrons (majorities) in the SCR. The corresponding value of $V_{s c}^{i n v}=$ $V_{t h} \ln \left(\frac{N_{D}}{n_{i}}\right)=0.88 \mathrm{~V}$ and thus $V_{r, R H E}^{i n v}=1.4 \mathrm{~V}$ are obtained. Therefore, a more detailed future model should take into account the electron continuity equation instead of assuming that the electron concentration upon illumination is equal to the electron concentration in the dark.
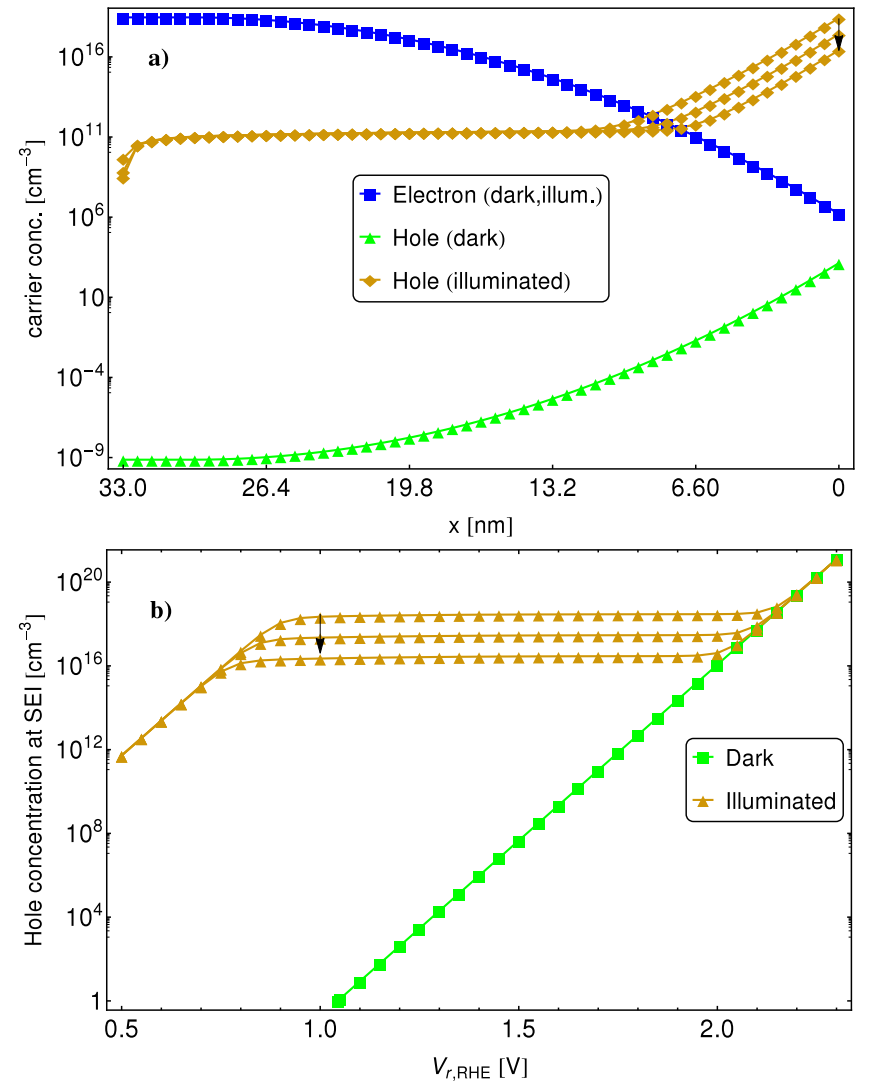

Figure 2: a) The simulated charge carrier concentrations in the semiconductor are shown for the measured voltage of $V_{r, R H E}=1.23 \mathrm{~V}$. Note the reverse orientation of the horizontal axis (also in Fig. 3 and Fig. 5) compared to Fig. 1. b) The hole concentration at the SEI as a function of $V_{r, R H E}$. The direction of arrows means increasing $k_{t r h}=10^{-4}, 10^{-3}, 10^{-2} \mathrm{~m} / \mathrm{s}$. The parameters for hematite from Table 2 were used. 
Upon illumination, the concentration of electrons is equal to the dark electron concentration. Fewer holes are accumulated near the SEI for increasing rate constant $k_{t r h}$ (faster charge transfer), Figure 2. For large $V_{r, R H E}(>2.0 \mathrm{~V})$, the hole concentration upon illumination near the SEI approaches the hole concentration in the dark, Figure 2b. At the back contact, the hole concentration follows from the solution of the continuity equation and the boundary condition in eq. 26 .

The energy band diagram is shown for a three-electrode measurement setup in Figure 3. The measured voltage of $V_{r, R H E}=1.23 \mathrm{~V}$ is assumed, which is a standard voltage used for the comparison of the different PEC electrodes. ${ }^{32,33}$ The measured voltage $V_{r, R H E}$ is indicated in Figure 3a) with an arrow on the energy scale, $-q V_{r, R H E}$. This notation is explained in our previous work. ${ }^{26}$ The band edges of the semiconductor $E_{c}(x), E_{v}(x)$ for the flatband condition $\left(V_{r, R H E}=V_{f b, R H E}\right)$ are shown as dashed lines, whereas those away from the flatband condition $\left(V_{r, R H E} \neq V_{f b, R H E}\right)$ are shown as solid lines. The band positions at the flatband conditions for hematite agree well with the values reported for $p H=1^{34,35}$ and $p H=14 .^{23}$ An upward band bending of the semiconductor is present if $V_{r, R H E} \mathrm{~V}$ is more positive than $V_{f b, R H E}$, see Figure 3. The band edges are pinned at the SEI by default (since we assume $V_{H}=V_{H}^{f b}$ ), but we allow for the modification of the surface conditions by changing the value of $V_{H}$ in our interactive band diagram software. ${ }^{21}$

The number of photogenerated electrons is small compared to the donor concentration, and thus the illumination does not change the electron concentration. Therefore, the electron quasi-Fermi level $E_{F n}$ is constant across the semiconductor, eq. 27 , and $E_{F n}=E_{F n, b}$. The position of $E_{F n}$ relative to $E_{0}^{R H E}$ in the energy diagram is given by the arrow $-q V_{r, R H E}$, eq. 8. In contrast, the hole concentration is determined mainly by photogenerated holes that are redistributed in the semiconductor according to the continuity equation Eq. 22. Since $E_{v}(0)$ is more positive than $E_{o x}$, the transfer of holes from the valence band can thermodynamically oxidize the electrolyte species. The external wire electrically connects the semiconductor to the metal counter electrode $(\mathrm{CE})$ through the potentiostat. The counter electrode Fermi 

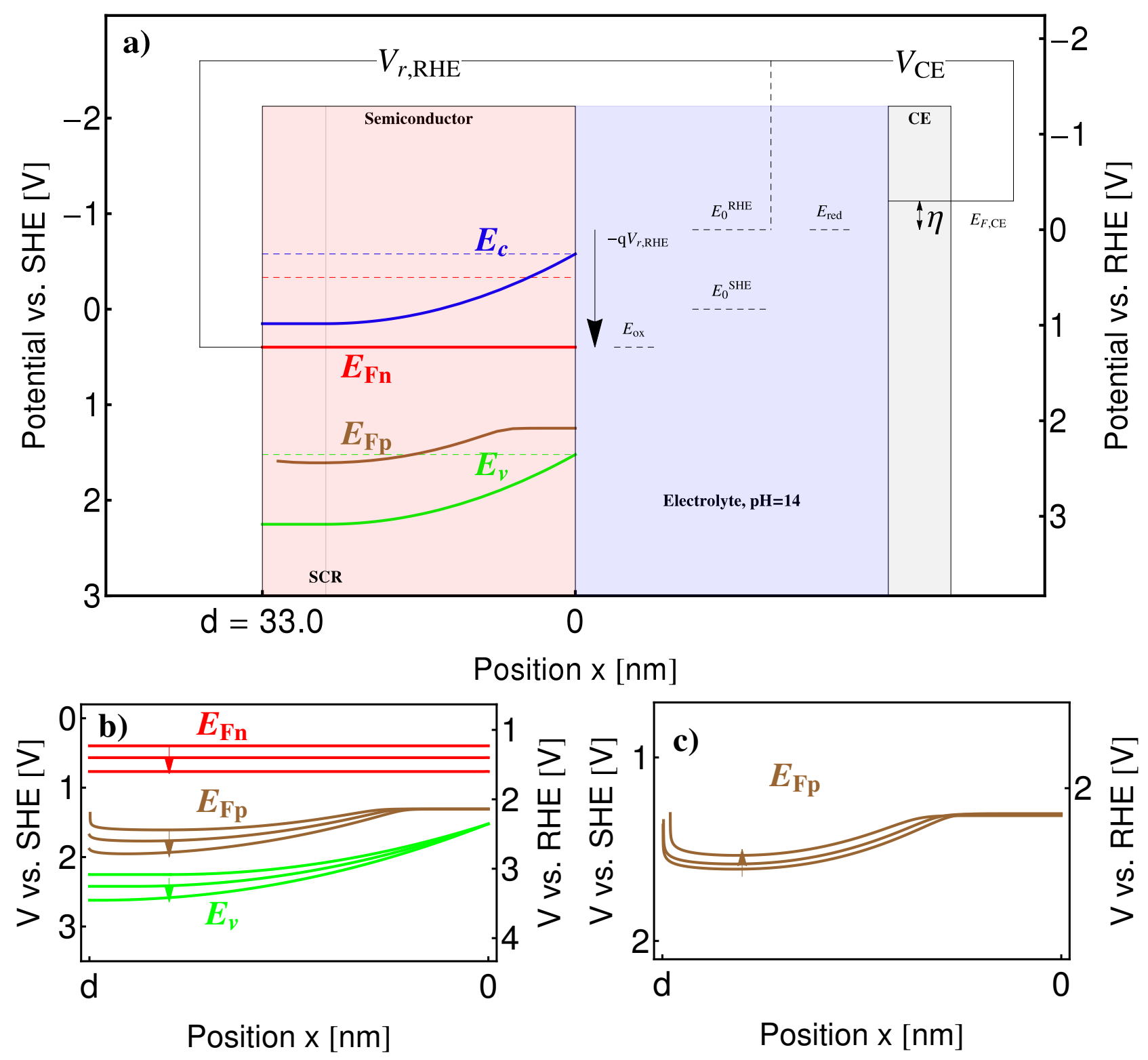

Figure 3: a) The calculated energy band diagram of the n-doped hematite at $V_{r, R H E}=1.23$ $\mathrm{V}$ and upon AM1.5G sunlight illumination. The semiconductor thickness is denoted $d$, the semiconductor-electrolyte interface is at $x=0 \mathrm{~nm}$, and the counter electrode is indicated by $\mathrm{CE}$ on the right hand side. The value of $k_{t r h}=10^{-3} \mathrm{~m} / \mathrm{s}$ is assumed for the rate constant of charge transfer of valence band holes to the electrolyte. Other material parameters are listed in Table 2. The interactive software tool to calculate the energy band diagram can be downloaded at http://icp.zhaw.ch/PEC. b) The quasi-Fermi level diagram, where the arrows indicate the increasing values of the measured voltage, $V_{r, R H E}=1.23,1.4,1.6 \mathrm{~V}$. c) The influence of the minority carrier diffusion length $L_{h}$ on the Quasi-Fermi level $E_{F_{p}}$ of holes, where the arrow indicates increasing values of $L_{h}=5,10,25 \mathrm{~nm}$. 
level $E_{F, C E}$ is automatically adjusted by applying the voltage $V_{C E}$ above the water reduction energy $E_{\text {red }}$ (including the electrochemical overpotential $\eta$ ) by the potentiostat to enable hydrogen evolution at the counter electrode. The counter electrode is shown in the energy diagram only to completely describe the three-electrode setup and we ignore its polarization in the following. ${ }^{36}$ In the electrolyte, we plot the two reference electrode energies $E_{0}^{S H E}$ and $E_{0}^{R H E}$, the standard water reduction and oxidation energy $E_{\text {red }}\left(0 \mathrm{eV}\right.$ vs RHE) and $E_{\text {ox }}(1.23$ $\mathrm{eV}$ vs RHE). Note that the relation of $E_{r e d}$ and $E_{o x}$ to $E_{r e d o x}$ depends on the concentrations (activities) of oxidizing and reducing species in the solution. ${ }^{37}$

The energy band diagram in the semiconductor for different values of the measured voltage $V_{r, R H E}$ is plotted in Figure $3 \mathrm{~b}$ ). For increasing $V_{r, R H E}$ the band bending increases and the electron quasi-Fermi level $E_{F n}$ shifts down on the RHE scale. Interestingly, the hole quasi-Fermi level $E_{F p}(0)$ at the SEI remains nearly constant for increasing $V_{r, R H E}$ (see Figure $\mathrm{S} 1$ in the Supporting Information) and thus the splitting of the quasi-Fermi levels approaches zero. In the neutral region $w<x<d$, the hole quasi-Fermi level $E_{F p}(x)$ is more negative for increasing $V_{r, R H E}$ and the photovoltage is nearly constant. When the hole diffusion length $L_{h}=\sqrt{D_{h} \tau_{h}}$ is increased, the flat region of the hole quasi-Fermi level $E_{F p}$ near the SEI is enlarged, Figure 3c), and the hole concentration in the neutral region decreases (see Figure S2 in the Supporting Information).

We simulated the photocurrent-voltage curves with our numerical model $j_{h}(0)$ (eq. 25) and compared the results with the published models of Gartner ${ }^{8}$ and Reichmann, ${ }^{10}$ Figure 4. According to the Gartner model, the minority charge carrier concentration is calculated from the diffusion equation, neglecting recombination in the SCR and assuming that every hole in SCR contributes to the photocurrent (infinitely fast hole/electron transfer to the electrolyte). The photocurrent density of Gartner is

$$
j_{G}=e P\left(1-\frac{e^{-\alpha w}}{1+\alpha L_{h}}\right) .
$$


Therefore, $j_{G}$ overestimates the minority carrier photocurrent in comparison to our numerical model $j_{h}(0)$. The recombination in the SCR by the Sah-Noyce-Shockley formalism was incorporated into the model by Reichmann ${ }^{10}$ with resulting photocurrent $j_{R}$ (the detailed expression is given in the Supporting Information). For small $V_{r, R H E}, j_{R}$ is much smaller than $j_{G}$ because the SCR recombination loss is included in $j_{R}$. The onset of the photocurrent calculated by Reichmann $j_{R}$ starts when $\gamma=\frac{j_{s} e^{-\frac{V_{a p p}}{V_{t h}}}}{q k_{t r h} p_{\text {dark }}(0)} \approx 1\left(j_{s}\right.$ is the saturation current density as defined in the SI). Therefore, if we consider faster charge transfer kinetics (larger $k_{t r h}$ ), we need a smaller value of the onset potential $V_{r, R H E}$ (and thus $V_{a p p}$ ) to obtain a similar value $\gamma \approx 1$. For increasing $V_{r, R H E}, j_{R}$ approaches $j_{G}$ because the SCR recombination becomes negligible in $j_{R},{ }^{10}$ but the numerical photocurrent $j_{h}(0)$ is smaller than $j_{G}$ since the SCR recombination is included in $j_{h}(0)$. The numerical photocurrent $j_{h}(0)$ onsets when $V_{r, R H E}$ is more positive than $V_{f b, R H E}$ and it is larger than $j_{R}$ for small $V_{r, R H E}$. Increasing the rate constant $k_{t r h}$ represents a faster exchange rate of holes with the solution. This also shifts the numerical $\mathrm{j}-\mathrm{V}$ curve to the left as predicted by the Reichmann model, decreasing the onset potential of the photocurrent.

The measured photocurrent-voltage (IV) responses of the nanostructured APCVD hematite in $\mathrm{H}_{2} \mathrm{O}_{2}{ }^{24}$ and $\mathrm{NaOH}^{38}$ electrolyte are compared with the prediction from our model in Figure 4. The IV profile from Ref. 24 appears similar to the Gartner model but shifted to lower photocurrents. In addition, the onset potential in Ref. 24 is about $0.1 \mathrm{~V}$ more negative than in the numerical model. Measurements in Ref. 38 were done on the electrode with $\mathrm{IrO}_{2}$ catalyst. The onset voltage $\approx 0.8 \mathrm{~V}_{R H E}$ of the measured photocurrent ${ }^{38}$ is approximately reproduced with the simulated photocurrent for the rate constant of $k_{t r h}=10^{-4} \mathrm{~m} / \mathrm{s}$. However, the slope of the measured photocurrent and its value $4.3 \mathrm{~mA} / \mathrm{cm}^{2}$ at $1.5 \mathrm{~V}_{r, R H E}$ are smaller than the slope of the simulated photocurrent and its value $3.8 \mathrm{~mA} / \mathrm{cm}^{2}$ at 1.5 $\mathrm{V}_{r, R H E}$.

These differences in the simulated and the measured photocurrent ${ }^{24,38}$ can be understood by discussing the assumptions of our model with respect to Ref. 24,38 , where the donor con- 
centration was roughly $N_{D} \approx 10^{20} \mathrm{~cm}^{-3}$, which is nearly two orders of magnitude larger than the donor concentration $N_{D}=2.91 \cdot 10^{18} \mathrm{~cm}^{-3}$ assumed in this work, Table 2. The main reason we chose parameters from Ref. 39 was the uniformity of the hematite film obtained (without nanostructures) and its constant thickness that corresponds well to our model. In addition, hematite in Ref. 24,38 is highly nanostructured (as compared to our compact film assumption) with varying thickness of the hematite cauliflower structures and increased light absorption due to the trapping of light in the nanostructure. Our model does not account for these effects and thus the validation of our model with IV measurements ${ }^{24,38}$ is not feasible. In addition, the IV response of the photoelectrode couples all physical processes with different time scales in a global photocurrent measurement. Thus disentangling of the individual processes from the IV response is difficult and it is usually achieved by spectroscopic methods ${ }^{40}$ which probe response of the system to spectrum of frequency perturbations.

We checked that the maximum photocurrent obtainable from the hematite electrode based purely on the number of absorbed photons is $q P=12.5 \mathrm{~mA} / \mathrm{cm}^{2}$ for AM1.5G illumination, which is the theoretical maximum based on the bandgap of hematite under these illumination conditions. This value is also obtained for the Gartner photocurrent eq. 29 when the bracket term is close to one and also for the Reichmann photocurrent (that recovers the Gartner photocurrent in regime of large voltages). The plateau of the numerical photocurrent $j_{h}(0)$ cannot be computed here, because our model cannot be used to predict photocurrents at voltages higher than $V_{r, R H E}>V_{r, R H E}^{i n v}$. At such voltages inversion layer is formed as described in the previous text and this would need degenerate statistics to be included in the model.

$\mathrm{Cu}_{2} \mathrm{O}$ We also applied our model to simulate charge transport in p-type semiconductors used as photocathodes. Appropriate changes in the equations were introduced, resulting from doping with acceptors rather than donors. Cuprous oxide $\left(\mathrm{Cu}_{2} \mathrm{O}\right)$ is an abundant and promising material for the PEC photocathodes. The main issue with $\mathrm{Cu}_{2} \mathrm{O}$ is its limited 


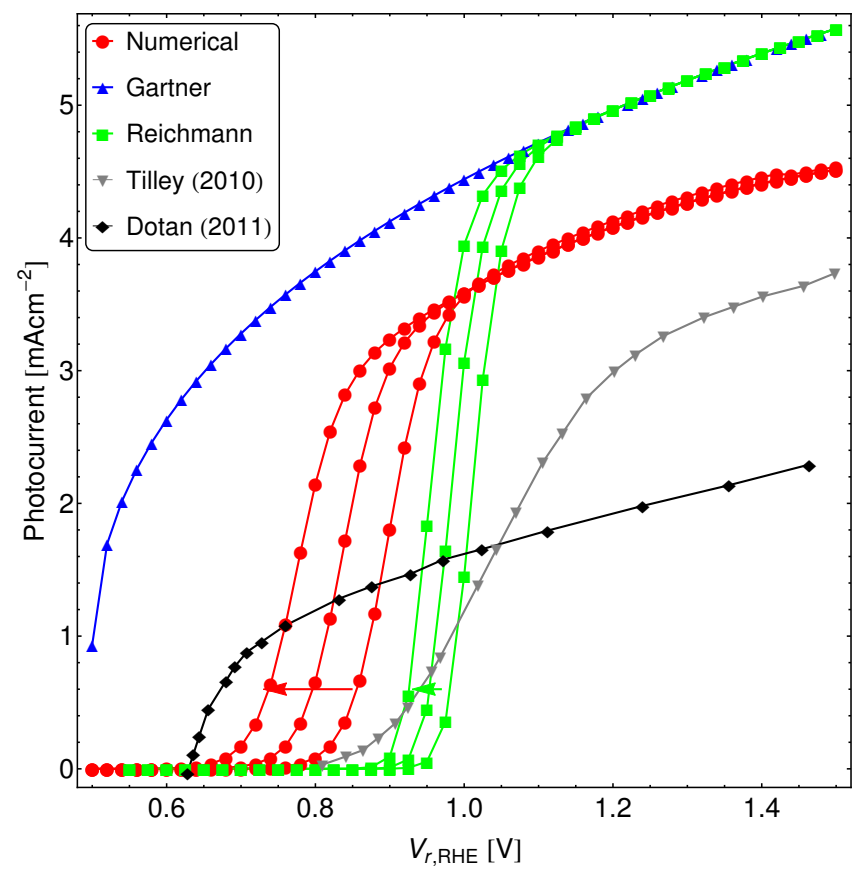

Figure 4: The photocurrent-voltage curves for $k_{t r h}=10^{-4}, 10^{-3}, 10^{-2} \mathrm{~m} / \mathrm{s}$ (in direction of arrows) from our numerical model, the Gartner model, the Reichmann model and the measured data from Tilley et al. ${ }^{38}$ and Dotan et al. ${ }^{24}$ for the n-doped hematite and other material parameters listed in Table 2.

stability in water that is currently being addressed with the stabilizing overlayers. ${ }^{41-43}$ The downward band-bending occurs when $V_{r, R H E}$ is more negative than $V_{f b, R H E}$. This leads to drift of electrons to the electrolyte, Figure 5. Upon illumination, the hole concentration is assumed to remain equal to the dark hole concentration. The electron concentration is calculated from the electron continuity equation. The electrons are accumulated near the SEI where they reduce water to $\mathrm{H}_{2}$ with the rate constant $k_{t r e}$.

In the case of p-type $\mathrm{Cu}_{2} \mathrm{O}$, the majority carriers are holes, and thus the counter electrode carries out the oxidation reaction (including the associated overpotential $\eta$ ). Although the electron quasi-Fermi level $E_{F n}$ is negative with respect to $E_{r e d}$, making it suitable for hydrogen evolution, Figure 5, corrosion prevents hydrogen evolution in the experiment unless the $\mathrm{Cu}_{2} \mathrm{O}$ is protected by overlayers. ${ }^{41}$ So far, our model does not consider corrosion; here we aimed at showing the general energetic configuration of the p-type PEC photoelectrode. 


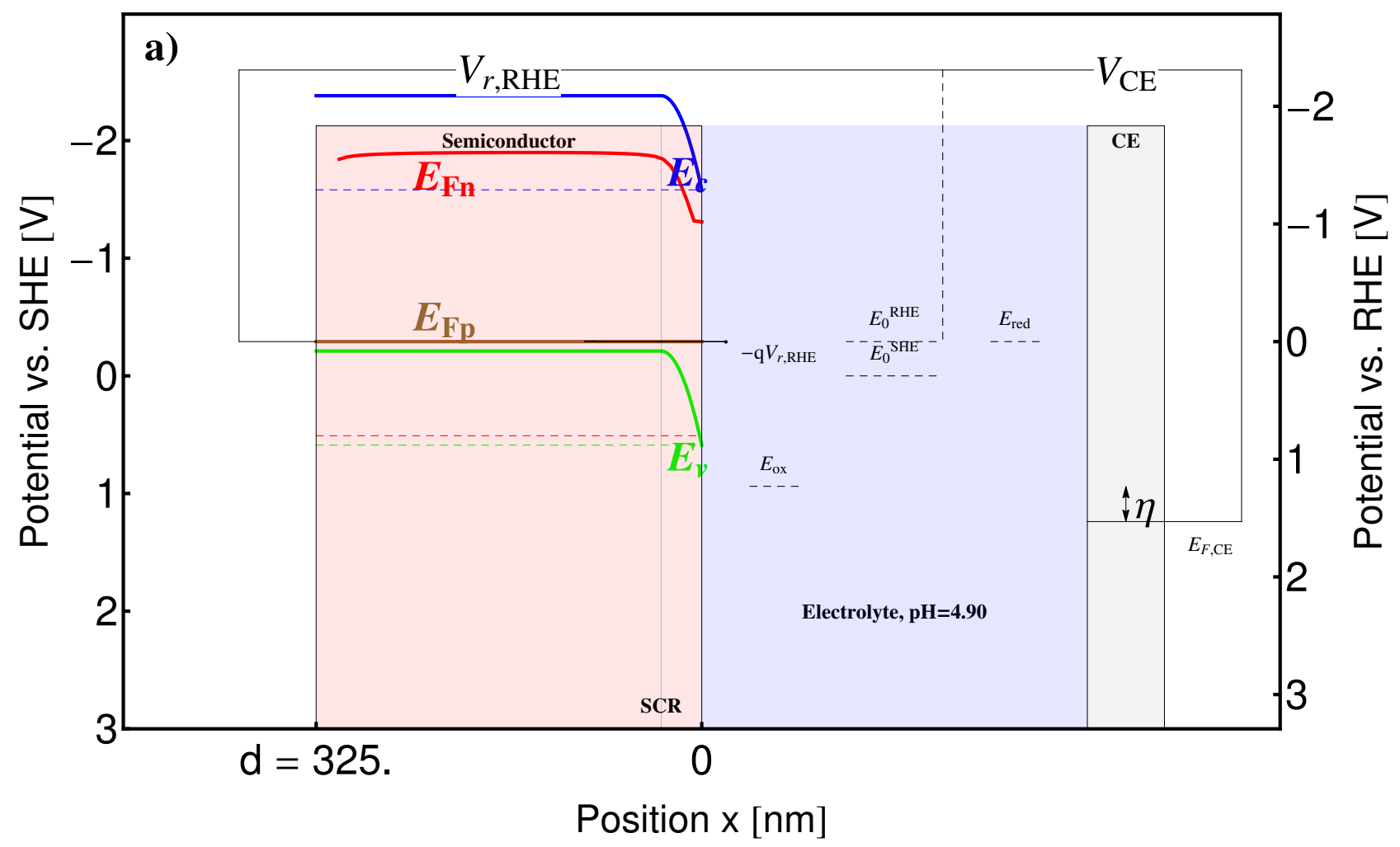

Figure 5: The calculated energy band diagram for the p-doped $\mathrm{Cu}_{2} \mathrm{O}, k_{t r e}=10 \mathrm{~m} / \mathrm{s}$ and $V_{r, R H E}=0 \mathrm{~V}$ (hence the voltage arrow is not visible in the diagram). The material parameters are listed in Table 2. The interactive tool for calculation of this figure can be accessed at http://icp.zhaw.ch/PEC. 
Conclusion We presented a physical model for minority charge carrier transport in semiconductor PEC electrodes in contact with an electrolyte. The direct charge transfer to the electrolyte from valence or conduction band, band-to-band recombination and Lambert-Beer optical generation were assumed. The numerical solution of the model equations allows us to calculate the minority carrier concentration and the quasi-Fermi level. Our resulting energy band diagram of the PEC cell accounts for the potential drop in the Helmholtz layer and it is capable of modeling both band edge pinning and unpinning. The differences in the simulated and measured photocurrent are due to the nanostructure effects on the charge transport and light absorption, which are not included in our model. The numerical model was implemented in the interactive software tool that can be freely accessed online. ${ }^{21}$ All presented results of this article can be reproduced with this software and we invite all members of the research community to use it while designing PEC cells. We are currently working on an extension of our model to a fully coupled drift-diffusion model with surface states. Such photoelectrode models need to accompany the experimental studies to suppress recombination losses (e.g. by the surface passivation) and enhance the charge transfer (e.g. by catalysis), the two major issues for efficient metal oxide photoelectrodes. ${ }^{44}$

\section{Acknowledgement}

We thank H. J. Lewerenz, G. Schlichthoerl, L. Bertoluzzi, F.T. Abdi, B. Klahr and A. Rothschild for fruitful discussions. The financial support by the Swiss Federal Office of Energy (PECHouse2 project, contract number SI/500090-02) is gratefully acknowleged.

\section{Supporting Information Available}

Equations for Reichmann photocurrent and additional simulation results are contained in Supporting Information. This information is available free of charge via the Internet at http://pubs.acs.org. This material is available free of charge via the Internet at http: //pubs.acs.org/. 


\section{References}

(1) Lewis, N. S.; Nocera, D. G. Powering the planet: Chemical challenges in solar energy utilization. Proceedings of the National Academy of Sciences 2006, 103, 15729-15735.

(2) Schiermeier, Q. Renewable power: Germany's energy gamble. Nature 2013, 496, 156158.

(3) Khaselev, O.; Turner, J. A. A Monolithic Photovoltaic-Photoelectrochemical Device for Hydrogen Production via Water Splitting. Science 1998, 280, 425-427.

(4) van de Krol, R.; Liang, Y. An n-Si/n-Fe2O3 Heterojunction Tandem Photoanode for Solar Water Splitting. CHIMIA International Journal for Chemistry 2013, 67, 168171.

(5) Sivula, K. Solar-to-Chemical Energy Conversion with Photoelectrochemical Tandem Cells. CHIMIA International Journal for Chemistry 2013, 67, 155-161.

(6) Abdi, F. F.; Han, L.; Smets, A. H. M.; Zeman, M.; Dam, B.; van de Krol, R. Efficient solar water splitting by enhanced charge separation in a bismuth vanadate-silicon tandem photoelectrode. Nature Communications 2013, 4.

(7) Krol, R. V. D.; Grätzel, M. Photoelectrochemical Hydrogen Production; Springer, 2011.

(8) Gärtner, W. W. Depletion-Layer Photoeffects in Semiconductors. Physical Review $1959,116,84-87$.

(9) Wilson, R. H. A model for the current-voltage curve of photoexcited semiconductor electrodes. Journal of Applied Physics 1977, 48, 4292-4297.

(10) Reichman, J. The current-voltage characteristics of semiconductor-electrolyte junction photovoltaic cells. Applied Physics Letters 1980, 36, 574-577. 
(11) Andrade, L.; Lopes, T.; Ribeiro, H. A.; Mendes, A. Transient phenomenological modeling of photoelectrochemical cells for water splitting - Application to undoped hematite electrodes. International Journal of Hydrogen Energy 2011, 36, 175-188.

(12) Foley, J. M.; Price, M. J.; Feldblyum, J. I.; Maldonado, S. Analysis of the operation of thin nanowire photoelectrodes for solar energy conversion. Energy \& Environmental Science 2012, 5, 5203-5220.

(13) Peter, L.; Li, J.; Peat, R. Surface recombination at semiconductor electrodes: Part I. Transient and steady-state photocurrents. Journal of Electroanalytical Chemistry and Interfacial Electrochemistry 1984, 165, 29-40.

(14) Klahr, B.; Gimenez, S.; Fabregat-Santiago, F.; Hamann, T.; Bisquert, J. Water Oxidation at Hematite Photoelectrodes: The Role of Surface States. J. Am. Chem. Soc. 2012, 134, 4294-4302.

(15) Bertoluzzi, L.; Bisquert, J. Equivalent Circuit of Electrons and Holes in Thin Semiconductor Films for Photoelectrochemical Water Splitting Applications. The Journal of Physical Chemistry Letters 2012, 2517-2522.

(16) Carver, C.; Ulissi, Z.; Ong, C.; Dennison, S.; Kelsall, G.; Hellgardt, K. Modelling and development of photoelectrochemical reactor for $\mathrm{H}_{2}$ production. International Journal of Hydrogen Energy 2012, 37, 2911-2923.

(17) Haussener, S.; Hu, S.; Xiang, C.; Weber, A. Z.; Lewis, N. Simulations of the irradiation and temperature dependence of the efficiency of tandem photoelectrochemical watersplitting systems. Energy $\&$ Environmental Science 2013,

(18) Salvador, P. Semiconductors' Photoelectrochemistry: A Kinetic and Thermodynamic Analysis in the Light of Equilibrium and Nonequilibrium Models. The Journal of Physical Chemistry B 2001, 105, 6128-6141. 
(19) Memming, R. Semiconductor Electrochemistry; John Wiley \& Sons, 2008.

(20) Peter, L. M. Energetics and kinetics of light-driven oxygen evolution at semiconductor electrodes: the example of hematite. Journal of Solid State Electrochemistry 2013, 17, $315-326$.

(21) Cendula, P. The model is available freely on the internet (accessed September 12, 2014). http://icp.zhaw.ch/PEC.

(22) Swierk, J. R.; Mallouk, T. E. Design and development of photoanodes for water-splitting dye-sensitized photoelectrochemical cells. Chemical Society Reviews 2013, 42, 23572387.

(23) Krol, R. v. d.; Liang, Y.; Schoonman, J. Solar hydrogen production with nanostructured metal oxides. J. Mater. Chem. 2008, 18, 2311-2320.

(24) Dotan, H.; Sivula, K.; Grätzel, M.; Rothschild, A.; Warren, S. C. Probing the photoelectrochemical properties of hematite $\left(\alpha-\mathrm{Fe}_{2} \mathrm{O}_{3}\right)$ electrodes using hydrogen peroxide as a hole scavenger. Energy $\&$ Environmental Science 2011, 4, 958.

(25) Klahr, B. M.; Hamann, T. W. Voltage dependent photocurrent of thin film hematite electrodes. Applied Physics Letters 2011, 99, 063508-063508-3.

(26) Bisquert, J.; Cendula, P.; Bertoluzzi, L.; Gimenez, S. Energy Diagram of Semiconductor/Electrolyte Junctions. The Journal of Physical Chemistry Letters 2013, 205-207.

(27) NREL, Solar Spectral Irradiance: Air Mass 1.5 (accessed March 17, 2012). http:// rredc.nrel.gov/solar/spectra/am1.5/.

(28) Tan, M. X.; Laibinis, P. E.; Nguyen, S. T.; Kesselman, J. M.; Stanton, C. E.; Lewis, N. S. In Progress in Inorganic Chemistry; Karlin, K. D., Ed.; John Wiley \& Sons, Inc., 1994; pp 21-144. 
(29) Markowich, P. A.; Ringhofer, C. A.; Schmeiser, C. Semiconductor equations; SpringerVerlag New York, Inc.: New York, NY, USA, 1990.

(30) Walter, M. G.; Warren, E. L.; McKone, J. R.; Boettcher, S. W.; Mi, Q.; Santori, E. A.; Lewis, N. S. Solar Water Splitting Cells. Chem. Rev. 2010, 110, 6446-6473.

(31) Mills, T. J.; Lin, F.; Boettcher, S. W. Theory and Simulations of Electrocatalyst-Coated Semiconductor Electrodes for Solar Water Splitting. Physical Review Letters 2014, 112, 148304.

(32) Kay, A.; Cesar, I.; Grätzel, M. New Benchmark for Water Photooxidation by Nanostructured $\mathrm{Fe}_{2} \mathrm{O}_{3}$ Films. J. Am. Chem. Soc. 2006, 128, 15714-15721.

(33) Chen, Z.; Deutsch, T. G.; Dinh, H. N.; Domen, K.; Emery, K.; Forman, A. J.; Gaillard, N.; Garland, R.; Heske, C.; Jaramillo, T. F. et al. Photoelectrochemical Water Splitting; SpringerBriefs in Energy; Springer New York, 2013; pp 7-16.

(34) Nozik, A. J. Photoelectrochemistry: Applications to Solar Energy Conversion. Annual Review of Physical Chemistry 1978, 29, 189-222.

(35) Grätzel, M. Photoelectrochemical cells. Nature 2001, 414, 338-344.

(36) Hodes, G. Photoelectrochemical Cell Measurements: Getting the Basics Right. The Journal of Physical Chemistry Letters 2012, 3, 1208-1213.

(37) Morrison, S. R. Electrochemistry at semiconductor and oxidized metal electrodes; Plenum Press, 1980.

(38) Tilley, S. D.; Cornuz, M.; Sivula, K.; Grätzel, M. Light-Induced Water Splitting with Hematite: Improved Nanostructure and Iridium Oxide Catalysis. Angewandte Chemie 2010, 122, 6549-6552. 
(39) Upul Wijayantha, K.; Saremi-Yarahmadi, S.; Peter, L. M. Kinetics of oxygen evolution at $\alpha-\mathrm{Fe}_{2} \mathrm{O}_{3}$ photoanodes: a study by photoelectrochemical impedance spectroscopy. Physical Chemistry Chemical Physics 2011, 13, 5264.

(40) Peter, L. Photoelectrochemical Water Splitting: Materials, Processes and Architectures; RSC Publishing, 2013.

(41) Paracchino, A.; Laporte, V.; Sivula, K.; Grätzel, M.; Thimsen, E. Highly active oxide photocathode for photoelectrochemical water reduction. Nat Mater 2011, 10, 456-461.

(42) Paracchino, A.; Mathews, N.; Hisatomi, T.; Stefik, M.; Tilley, S. D.; Grätzel, M. Ultrathin films on copper(i) oxide water splitting photocathodes: a study on performance and stability. Energy \&6 Environmental Science 2012, 5, 8673.

(43) Tilley, S. D.; Schreier, M.; Azevedo, J.; Stefik, M.; Graetzel, M. Ruthenium Oxide Hydrogen Evolution Catalysis on Composite Cuprous Oxide Water-Splitting Photocathodes. Advanced Functional Materials 2014, 24, 303-311.

(44) Sivula, K. Metal Oxide Photoelectrodes for Solar Fuel Production, Surface Traps, and Catalysis. The Journal of Physical Chemistry Letters 2013, 4, 1624-1633.

(45) Paracchino, A.; Brauer, J. C.; Moser, J.-E.; Thimsen, E.; Graetzel, M. Synthesis and Characterization of High-Photoactivity Electrodeposited $\mathrm{Cu}_{2} \mathrm{O}$ Solar Absorber by Photoelectrochemistry and Ultrafast Spectroscopy. The Journal of Physical Chemistry C 2012, 116, 7341-7350.

(46) Xu, Y.; Schoonen, M. A. A. The absolute energy positions of conduction and valence bands of selected semiconducting minerals. American Mineralogist 2000, 85, 543-556.

(47) Niu, M.; Huang, F.; Cui, L.; Huang, P.; Yu, Y.; Wang, Y. Hydrothermal Synthesis, Structural Characteristics, and Enhanced Photocatalysis of $\mathrm{SnO}_{2} / \alpha-\mathrm{Fe}_{2} \mathrm{O}_{3}$ Semiconductor Nanoheterostructures. ACS Nano 2010, 4, 681-688. 
(48) Morin, F. J. Electrical Properties of $\alpha-\mathrm{Fe}_{2} \mathrm{O}_{3}$. Physical Review 1954, 93, 1195-1199.

(49) Cesar, I.; Sivula, K.; Kay, A.; Zboril, R.; Grätzel, M. Influence of Feature Size, Film Thickness, and Silicon Doping on the Performance of Nanostructured Hematite Photoanodes for Solar Water Splitting. J. Phys. Chem. C 2008, 113, 772-782.

(50) Glasscock, J. A.; Barnes, P. R. F.; Plumb, I. C.; Bendavid, A.; Martin, P. J. Structural, optical and electrical properties of undoped polycrystalline hematite thin films produced using filtered arc deposition. Thin Solid Films 2008, 516, 1716-1724.

(51) Bosman, A.; van Daal, H. Small-polaron versus band conduction in some transitionmetal oxides. Advances in Physics 1970, 19, 1-117. 


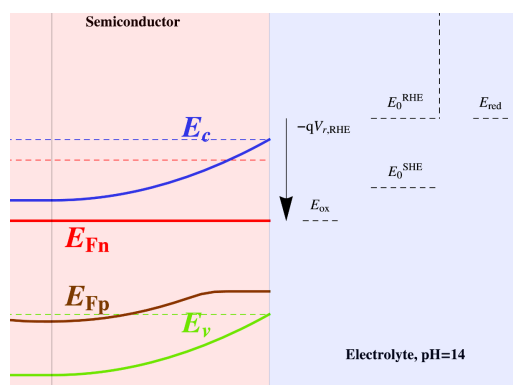

Figure 6: TOC graphic 

Table 1: Table of symbols and abbreviations. Symbols for material parameters
are defined in Table 2 .

\begin{tabular}{|c|c|c|}
\hline Symbol & Unit & Description \\
\hline $\mathrm{CE}$ & & Counter electrode \\
\hline LVL & & Local vacuum level \\
\hline PEC & & Photoelectrochemical \\
\hline SCR & & Space-charge region \\
\hline SEI & & Semiconductor-electrolyte interface \\
\hline SHE & & Standard hydrogen electrode \\
\hline SI & & Supporting information \\
\hline RHE & & Reversible hydrogen electrode \\
\hline Subscript $i$ & & Quantity in the isolated semiconductor before contact to an electrolyte \\
\hline Subscript b & & Quantity in the semiconductor bulk \\
\hline Subscript s & & Quantity at the SEI \\
\hline$k_{B}$ & $\mathrm{eV} / \mathrm{K}$ & Boltzmann constant $\left(8.6 \cdot 10^{-5} \mathrm{eV} / \mathrm{K}\right)$ \\
\hline$T$ & & Temperature $(300 \mathrm{~K})$ \\
\hline$q$ & $\mathrm{C}$ & Elementary charge $\left(1.6 \cdot 10^{-19} \mathrm{C}\right)$ \\
\hline$V_{t h}$ & $\mathrm{~V}$ & Thermal voltage $(25.9 \mathrm{mV})$ \\
\hline$h$ & $\mathrm{~J} \cdot \mathrm{s}$ & Planck's constant $\left(6.62607 \cdot 10^{-34} \mathrm{~J} \cdot \mathrm{s}\right)$ \\
\hline$c$ & $\mathrm{~m} / \mathrm{s}$ & Speed of light in vacuum $(299792458 \mathrm{~m} / \mathrm{s})$ \\
\hline$V_{r}$ & $\mathrm{~V}$ & Measurable voltage with respect to SHE reference electrode \\
\hline$V_{r, R H E}$ & $\mathrm{~V}$ & Measurable voltage with respect to RHE \\
\hline$V_{r, R H E}^{i n v}$ & $\mathrm{~V}$ & Measurable voltage with respect to RHE when the inversion layer starts to form \\
\hline$V_{f b}$ & $\mathrm{~V}$ & Flatband voltage with respect to SHE \\
\hline$V_{f b, R H E}$ & $\mathrm{~V}$ & Flatband voltage with respect to RHE \\
\hline$V_{a p p}$ & $\mathrm{~V}$ & Applied voltage to the semiconductor with respect to the dark equilibrium (unbiased) \\
\hline$V_{H}$ & $\mathrm{~V}$ & Potential (voltage) drop across the Helmholtz layer in the dark \\
\hline$V_{H}^{f b}$ & $\mathrm{~V}$ & Potential (voltage) drop across the Helmholtz layer at flatband situation in the dark \\
\hline$V_{H 0}^{n}$ & $\mathrm{~V}$ & Potential (voltage) drop across the Helmholtz layer in the dark equilibrium \\
\hline$V_{s c}$ & $\mathrm{~V}$ & Potential (voltage) drop across the semiconductor \\
\hline$V_{c s}$ & $\mathrm{~V}$ & Potential of the conduction band at the SEI \\
\hline$V_{b i}$ & $\mathrm{~V}$ & Built-in voltage of semiconductor/liquid junction \\
\hline$V_{C E}$ & $\mathrm{~V}$ & Voltage between the reference electrode and counterelectrode \\
\hline$\eta$ & $\mathrm{V}$ & Electrochemical overpotential at the CE \\
\hline$E_{v a c}$ & $\mathrm{eV}$ & Energy of the local vacuum level \\
\hline$E_{0}^{S H E}$ & $\mathrm{eV}$ & Energy of the SHE with respect to vacuum level of the electron $(-4.44 \mathrm{eV})$ \\
\hline$E_{0}^{R H E}$ & $\mathrm{eV}$ & Energy of the RHE with respect to vacuum level of the electron \\
\hline$E_{\text {redox }}$ & $\mathrm{eV}$ & Fermi level of the electrolyte species (redox level) \\
\hline$E_{\text {red }}$ & $\mathrm{eV}$ & Standard water reduction energy \\
\hline$E_{o x}$ & $\mathrm{eV}$ & Standard water oxidation energy \\
\hline$E_{F 0}$ & $\mathrm{eV}$ & Equilibrium Fermi level in the semiconductor (dark) \\
\hline$E_{c, 0 i}$ & $\mathrm{eV}$ & Conduction band edge in the isolated semiconductor before contact to an electrolyte \\
\hline$E_{F, 0 i}$ & $\mathrm{eV}$ & Fermi level in the isolated semiconductor before contact to an electrolyte \\
\hline$E_{F n}, E_{F p}$ & $\mathrm{eV}$ & Quasi-Fermi energy of electrons and holes \\
\hline$E_{F n, b}$ & $\mathrm{eV}$ & Quasi-Fermi energy of electrons at the back contact \\
\hline & $\mathrm{eV}$ & Conduction band edge in the semiconductor \\
\hline$E_{c s}$ & $\mathrm{eV}$ & Conduction band edge at the SEI \\
\hline$E_{v}$ & $\mathrm{eV}$ & Valence band edge in the semiconductor \\
\hline$E_{v s}$ & $\mathrm{eV}$ & Valence band edge at the SEI \\
\hline$E_{F, C E}$ & $\mathrm{eV}$ & Fermi level of the CE \\
\hline$\zeta_{n b}$ & $\mathrm{eV}$ & The difference between the semiconductor conduction band energy and the electron Fermi level \\
\hline
\end{tabular}


Table 1 continuted.

\begin{tabular}{|l|l|l|}
\hline Symbol & Unit & Description \\
\hline$\phi$ & $\mathrm{V}$ & Local electrostatic potential \\
$\phi_{a}$ & $\mathrm{~V}$ & Approximate solution for local electrostatic potential \\
$\phi_{e l}$ & $\mathrm{~V}$ & Local electrostatic potential of the electrolyte \\
$\phi_{s}$ & $\mathrm{~V}$ & Local electrostatic potential at SEI \\
$\phi_{b}$ & $\mathrm{~V}$ & Local electrostatic potential in the semiconductor bulk \\
$n_{i}$ & $\mathrm{~m}^{-3}$ & Intrinsic carrier concentration in the bulk of semiconductor \\
$n_{0 i}, p_{0 i}$ & $\mathrm{~m}^{-3}$ & Equilibrium concentration of electrons and holes in the bulk of isolated semiconductor \\
$n_{\text {dark }}, p_{d a r k}$ & $\mathrm{~m}^{-3}$ & Dark concentration of electrons and holes \\
$n, p$ & $\mathrm{~m}^{-3}$ & Concentration of electrons and holes \\
$w$ & $\mathrm{~m}$ & Width of the space-charge region in the semiconductor \\
$j_{h}$ & $\mathrm{~A} / \mathrm{m}^{2}$ & Hole current density \\
$j_{G}$ & $\mathrm{~A} / \mathrm{m}^{2}$ & Photocurrent density calculated by Gartner8 \\
$j_{R}$ & $\mathrm{~A} / \mathrm{m}^{2}$ & Photocurrent density calculated by Reichmann \\
$j_{s}$ & $\mathrm{~A} / \mathrm{m}^{2}$ & Saturation current density \\
$G_{h}, R_{h}$ & $\mathrm{~m}^{-3} \mathrm{~s}^{-1}$ & Generation and recombination rate of holes \\
$P$ & $\mathrm{~m}^{-2} \mathrm{~s}^{-1}$ & Number of photons absorbed in the semiconductor from AM1.5G spectrum \\
$\Phi$ & $\mathrm{m}^{-3} \mathrm{~s}^{-1}$ & Spectral photon flux of AM1.5G spectrum \\
$\mu_{h}$ & $\mathrm{~m}^{2} \mathrm{~V}^{-1} \mathrm{~s}^{-1}$ & Mobility of holes \\
$D_{h}$ & $\mathrm{~m}^{2} \mathrm{~s}^{-1}$ & Diffusion constant of holes \\
$k_{t r h}$ & $\mathrm{~ms}^{-1}$ & Rate constant for charge transfer of VB holes to electrolyte \\
$\lambda_{g}$ & $\mathrm{~m}^{-1}$ & Wavelength below which semiconductor absorbs photons \\
$r_{s}$ & $\mathrm{~ms}^{-1}$ & Back contact surface recombination velocity \\
\hline
\end{tabular}

Table 2: Material parameters of semiconductors used in the calculations.

\begin{tabular}{|l|l|l|l|}
\hline Symbol & $\mathrm{Fe}_{2} \mathrm{O}_{3}{ }^{39}$ & $\mathrm{Cu}_{2} \mathrm{O}^{42,45}$ & Description \\
\hline$N_{D}\left[\mathrm{~cm}^{-3}\right]$ & $2.91 \cdot 10^{18}$ & 0 & Donor concentration \\
$N_{A}\left[\mathrm{~cm}^{-3}\right]$ & 0 & $5 \cdot 10^{17}$ & Acceptor concentration \\
$V_{f b, R H E}[\mathrm{~V}]$ & +0.5 & +0.8 & Flatband potential \\
$\chi[\mathrm{eV}]$ & $+4.78^{46,47}$ & $+4.22^{46}$ & Electron affinity \\
$N_{C}\left[\mathrm{~cm}^{-3}\right.$ & $4 \cdot 10^{22} 48,49$ & $1.1 \cdot 10^{19}$ & Density of states of CB \\
$N_{V}\left[\mathrm{~cm}^{-3}\right]$ & $1 \cdot 10^{22}$ & $1.1 \cdot 10^{19}$ & Density of states of VB \\
$\varepsilon_{r}$ & $32^{50}$ & 6.6 & Relative permitivity \\
$E_{g}[\mathrm{eV}]$ & 2.1 & 2.17 & Bandgap energy \\
$d[\mathrm{~nm}]$ & 33 & 325 & Thickness of semiconductor \\
$\tau_{e}[\mathrm{~ns}]$ & - & 0.25 & Electron lifetime \\
$\tau_{h}[\mathrm{~ns}]$ & $0.048^{51}$ & - & Hole lifetime \\
$L_{e}[\mathrm{~nm}]$ & - & 40 & Electron diffusion length \\
$L_{h}[\mathrm{~nm}]$ & $5^{7}$ & - & Hole diffusion length \\
$\alpha\left[\mathrm{cm}{ }^{-1}\right]$ & $1.5 \cdot 10^{5}$ & $1.3 \cdot 10^{4}$ & Absorption coefficient \\
$p H$ & 14 & 4.9 & pH value of the electrolyte \\
\hline
\end{tabular}

